\title{
Primer registro de Piriqueta mexicana (Passifloraceae) para la flora de Chiapas, México
}

\author{
First record of Piriqueta mexicana (Passifloraceae) for \\ the flora of Chiapas, Mexico
}

\section{Acta Botanica Mexicana}

\author{
Diana Yaneth Sánchez-Molina!’3 (iD), Ana Laura Gómez-Pérez² (iD y Neptalí Ramírez-Marcial' (D)
}

\section{Resumen:}

Antecedentes y Objetivos: La flora de Chiapas es una de las más diversas de México y aún existen especies que no se han descrito o registrado. El objetivo de este estudio es dar a conocer la presencia de Piriqueta mexicana colectada en el Área Natural Protegida "La Concordia Zaragoza".

Métodos: Se colectaron ejemplares botánicos de Piriqueta, los cuales se determinaron taxonómicamente mediante el uso de claves especializadas, consulta de material en colecciones virtuales y con taxónomos expertos. Además, se realizaron mediciones morfométricas de peciolo, hojas, flores, frutos y semillas del material colectado y se compararon con las descripciones del ejemplar tipo y de revisiones taxonómicas.

Resultados clave: Se confirma la presencia de Piriqueta mexicana para la flora de Chiapas y con este nuevo registro se amplía a dos el número de especies de Piriqueta presentes en esta entidad.

Conclusiones: Se amplía el área de distribución de Piriqueta mexicana y se reafirma la importancia de continuar con exploraciones botánicas en las Áreas Naturales Protegidas, las cuales representan reservorios efectivos para mantener especies poco conocidas.

Palabras clave: área natural protegida, colectas botánicas, nuevo registro.

\section{Abstract:}

Background and Aims: The flora of Chiapas is one of the most diverse in Mexico, but there are still species that have not been described or registered. The objective of this study is to publish the presence of Piriqueta mexicana collected in the Protected Natural Area "La Concordia Zaragoza".

Methods: Botanical specimens of Piriqueta were collected and were determined taxonomically through the use of specialized keys, consultation of material in virtual collections and with expert taxonomists. In addition, morphometric measurements of petiole, leaves, flowers, fruits and seeds of the collected material were compared with the descriptions of the type specimen and taxonomic revisions.

Key results: The presence of Piriqueta mexicana is confirmed for the flora of Chiapas; the number of Piriqueta species present in this entity is expanded to two.

Conclusions: The distribution area of Piriqueta mexicana extends to southern Mexico and this finding reaffirms the importance of continuing with botanical explorations in Protected Natural Areas, which represent effective reservoirs to maintain poorly known species.

Key words: botanical collections, new record, protected natural area.

${ }^{1}$ El Colegio de la Frontera Sur (ECOSUR), Departamento de Conservación de la Biodiversidad, 29290 San Cristóbal de las Casas, Chiapas, México.

"Dirección del Jardín Botánico "Dr. Faustino Miranda", Curaduría General de Flora (SEMAHN), 29000 Tuxtla Gutiérrez, Chiapas, México.

${ }^{3}$ Autor para la correspondencia: diana.sanchez@ecosur.mx
Recibido: 24 de febrero de 2020.

Revisado: 20 de marzo de 2020.

Aceptado por Marie-Stéphanie Samain: 7 de abril de 2020

Publicado Primero en línea: 16 de abril de 2020. Publicado: Acta Botanica Mexicana 127 (2020).
Citar como:

Sánchez-Molina, D. Y., A. L. Gómez-Pérez y N. Ramírez-Marcial. Primer registro de Piriqueta mexicana (Passifloraceae) para la flora de Chiapas, México. Acta Botanica Mexicana 127: e1684. DOI: 10.21829/ abm127.2020.1684 


\section{Introducción}

El estado de Chiapas alberga una alta diversidad florística (Breedlove, 1981; 1986). El número de plantas vasculares nativas reconocidas para Chiapas es de 8790 especies (Villaseñor, 2016). No obstante, en los últimos años se han registrado y descrito nuevos taxones, por lo que este número va en aumento (p. ej., Ortiz-Díaz et al., 2016; Martínez-Meléndez et al., 2017; García-Martínez et al., 2018; Areces-Berazain y Vega-López, 2019; Dean et al., 2019; Lozada-Pérez, 2019; RuizSánchez et al., 2019; Torres-Colín y Alvarado-Cárdenas, 2019; Vicente-Rivera et al., 2019). Varios de los registros recientemente descritos han sido encontrados en Áreas Naturales Protegidas dentro del territorio estatal y es posible que a medida que se incremente el esfuerzo de muestreo también se detecten otros previamente no examinados.

El estado cuenta con 37 Áreas Naturales Protegidas de control estatal y federal (INAFED, 2010). La continua transformación de estos paisajes naturales representa amenazas directas para la conservación de la flora y fauna de Chiapas (González-Espinosa et al., 2005). A pesar de lo anterior, en algunas de estas áreas protegidas, la heterogeneidad de hábitats y el acceso controlado representan refugios efectivos para la permanencia de especies poco conocidas, como es el caso de Piriqueta mexicana Fryxell \& S.D. Koch.

A la fecha, ningún estudio publicado había registrado la presencia de Piriqueta mexicana como parte de la flora de Chiapas (Breedlove, 1981; Arbo, 1995; Lozada, 2010; Villaseñor, 2016). La revisión de algunas colectas botánicas obtenidas recientemente en el Área Natural Protegida "La Concordia Zaragoza", ubicada en la Depresión Central de Chiapas, sugiere la existencia de esta especie en Chiapas.

En este estudio, se presenta información que confirma el registro de Piriqueta mexicana en el estado de Chiapas. Dicha especie parece tener una distribución restringida a un Área Natural Protegida del estado, se discuten sus similitudes y diferencias con los ejemplares tipo de la especie, y su descripción taxonómica.

\section{Materiales y Métodos}

El estudio se realizó en el Área Natural Típica "La Concordia Zaragoza", municipio La Concordia, ubicada en la Depresión Central de Chiapas. Esta región se sitúa entre $16^{\circ} 03^{\prime}$ y $16^{\circ} 55^{\prime}$ de latitud norte y $91^{\circ} 52^{\prime}$ y $93^{\circ} 44^{\prime}$ de longitud oeste
(Reyes-García y Sousa, 1997). Es una extensa zona semiplana circundada por la Sierra Madre de Chiapas, la Altiplanicie Central y las Montañas del Norte (INAFED, 2010). La vegetación original incluye diversas asociaciones de selva baja caducifolia por debajo de 800 m s.n.m., selva mediana en altitudes superiores a 800 m s.n.m. y bosques de encinos entre 800 y 1500 m s.n.m. (INAFED, 2010; Rocha-Loredo et al., 2010).

A partir de varias exploraciones botánicas y estudios ecológicos llevados a cabo entre 2017 y 2018 en esta Área Natural Protegida, se realizaron colectas botánicas siguiendo la metodología propuesta por Lot y Chiang (1986). Se obtuvieron medidas morfométricas de estructuras que contribuyen a su identificación (hojas, peciolos, flores, frutos y semillas). Para su determinación taxonómica se utilizaron claves especializadas para Passifloraceae y Turneraceae (Arbo, 1995; Lozada, 2010) y se consultó a la Dra. Ma. Mercedes Arbo del Instituto de Botánica del Nordeste CONICET (UNNE, Argentina), especialista en la familia Turneraceae (ahora Passifloraceae). Los resultados de las mediciones fueron cotejados con lo reportado en la literatura y se hicieron comparaciones con ejemplares digitalizados disponibles en las páginas electrónicas de JSTOR Global Plants (JSTOR, 2019), Missouri Botanical Garden (TROPICOS, 2019), Global Biodiversity Information Facility (GBIF, 2019), el Portal de Datos Abiertos UNAM (IBUNAM, 2019), y la base de datos del SEINet (SEINEt, 2019). Asimismo, se consultaron los registros del Sistema Nacional sobre Biodiversidad (CONABIO, 2018). Con la información de todos los registros recopilados, se elaboró un mapa de distribución de dicha especie en México (Fig. 1).

Los ejemplares colectados en Chiapas fueron depositados en el herbario de la Secretaría de Medio Ambiente e Historia Natural (CHIP) y el Herbario Eizi Matuda (HEM) de la Universidad de Ciencias y Artes de Chiapas. Otros duplicados serán distribuidos al Herbario Nacional de México (MEXU) y al herbario de la Universidad de Texas en Austin (TEX).

\section{Resultados}

Piriqueta mexicana Fryxell \& S.D. Koch, Aliso 11(4): 558-560, f. 10.1987. 


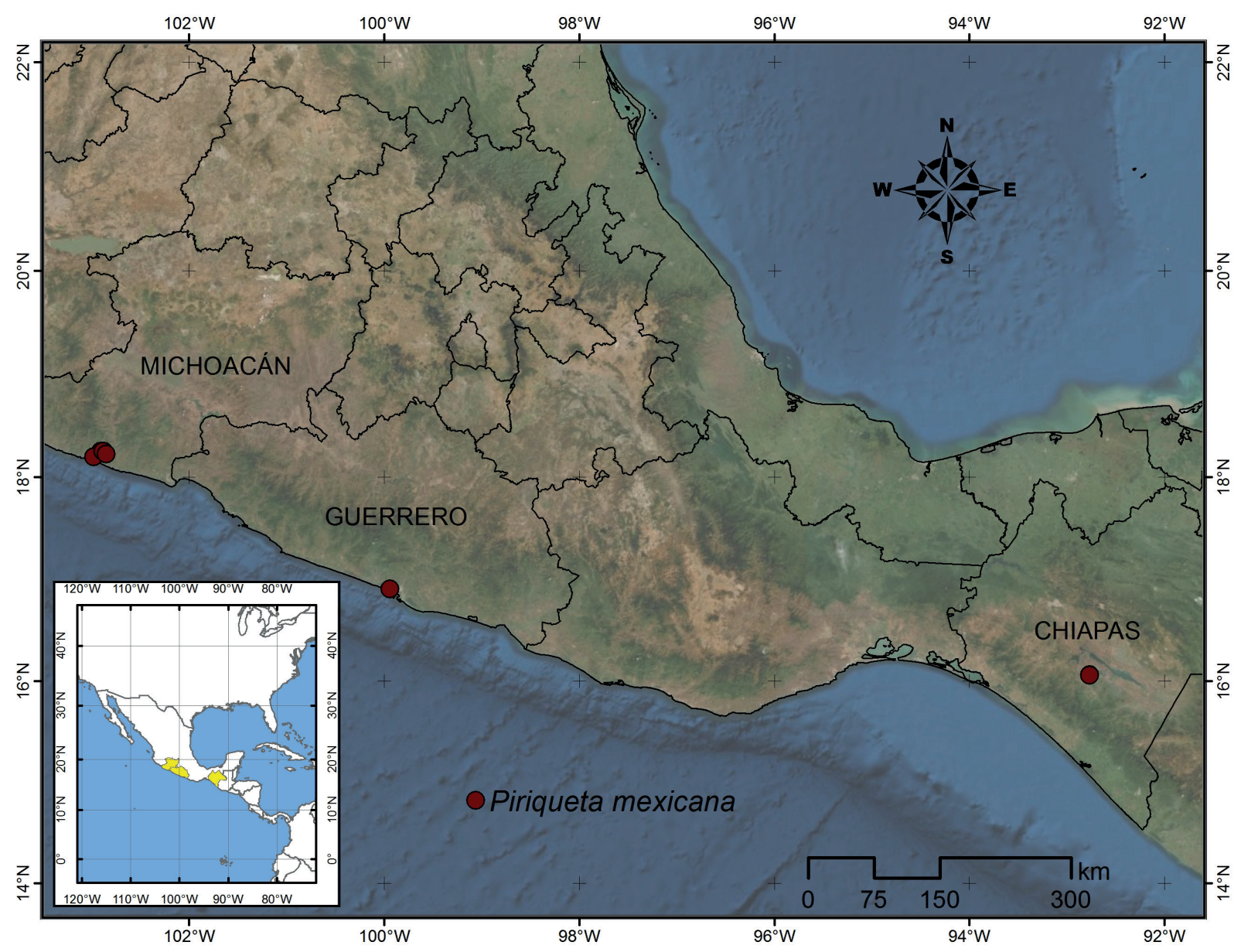

Figura 1: Mapa de distribución de Piriqueta mexicana Fryxell \& S.D. Koch en México con base en registros de colectas históricas.

TIPO: MÉXICO. Michoacán, municipio Aquila, Terracería a la mina "Varicosta", $13 \mathrm{~km}$ al $\mathrm{N}$ de la carretera Costera $(73$ $\mathrm{km}$ al $\mathrm{O}$ de playa Azul), $750 \mathrm{~m}, 18^{\circ} 13^{\prime} 40^{\prime \prime} \mathrm{N}, 102^{\circ} 51^{\prime} 18^{\prime \prime} \mathrm{O}$, 18.XI.1983, S. D. Koch y P. A. Fryxell 83190 (holotipo: CHAPA!, isotipos: CAS!, CTES!, ENCB!, F!, MEXU!, MO!, NY!, RSA!, TEX!, US!).

Los ejemplares de Piriqueta Aubl. colectados en Chiapas corresponden a P. mexicana (Fig. 2), aunque presentan un porte más bajo a lo reportado en el ejemplar tipo (Fig. 2A); coinciden en la presencia de tricomas glandulares y porrecto-estrellados con brazos cortos en el taIlo; pelos glandulares en el borde de las hojas, tricomas laxos y porrecto-estrellados en el haz y tricomas estrellados en el envés; flores axilares, solitarias, homostilas, pétalos cinco de color durazno con líneas oscuras (Figs. 2B, C); frutos ovoides 4-7 mm de largo, con pelos glandulares en el exterior, lisos por dentro (Fig. 2D); semillas obovoides de $2 \times 1 \mathrm{~mm}$, de color blanco o crema que se tornan a café cuando maduran (Fig. 2E).

Hábitat: la población observada en Chiapas incluye pocos individuos dispersos en un área no mayor a $5 \mathrm{~km}^{2}$. Está presente predominantemente en condiciones de vegetación secundaria, derivada del bosque tropical caducifolio entremezclado con vegetación remanente de bosque de Quercus acutifolia Née, Q. elliptica Née, Q. sapotifolia Liebm. y $Q$. segoviensis Liebm. Otros individuos fueron observados en vegetación secundaria en fragmentos de bosque tropical subcaducifolio y bosque de Pinus oocarpa Schiede ex Schltdl. y P. maximinoi H.E. Moore, en un rango de altitud entre 530 y $780 \mathrm{~m}$. Por lo general, se observó en áreas planas, sobre suelos medianamente profundos de color rojo y amarillo claro. El monitoreo frecuente permi- 


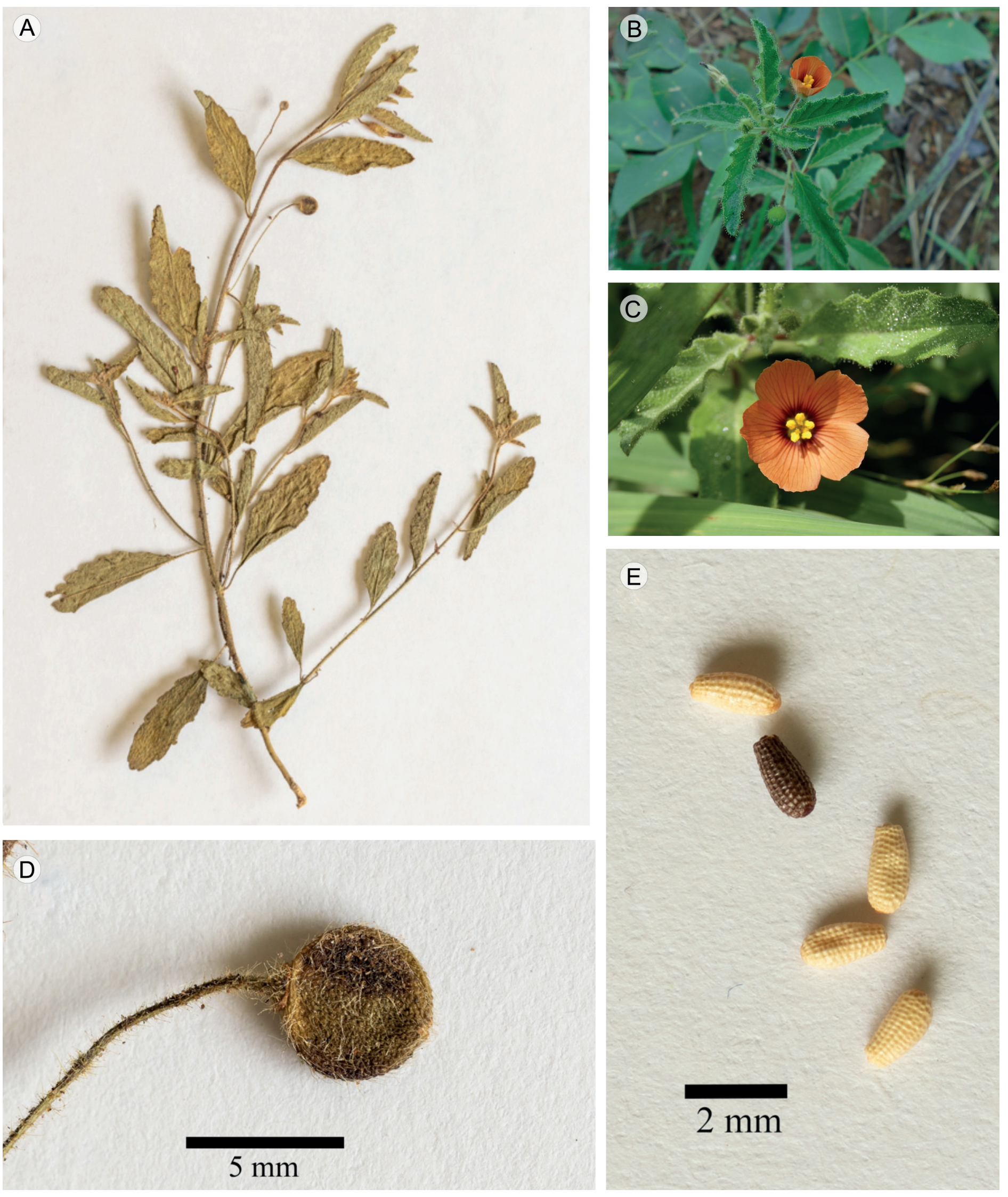

Figura 2: Aspecto general de Piriqueta mexicana Fryxell \& S.D. Koch en Chiapas, México: A. planta completa; B-C. hojas y flor; D. fruto; E. semillas. Fotografías: L. E. Gómez-Pérez (A, D y E), M. A. García Jiménez (B) y D. C. Gallegos López (C). 
tió observar que la especie florece y fructifica de junio a septiembre. No se conocen usos para esta especie.

Ejemplares examinados: MÉXICO. Chiapas, municipio La Concordia, ejido Nueva Libertad, $548 \mathrm{~m}, 16^{\circ} 03^{\prime} 53^{\prime \prime} \mathrm{N}$, 9246'10"O, 18.VIII.2017, D. Y. Sánchez-Molina 1056 (CHIP, HEM); loc. cit., 553 m, 1603'53"N, 9246'09"O, 22.VIII.2018, D. Y. Sánchez-Molina 1136 (CHIP, HEM).

\section{Discusión}

En México se tienen registradas tres especies de Piriqueta: P. mexicana, P. cistoides (L.) Griseb. y P. mortonii S.D. Koch \& Fryxell, de las cuales hasta ahora sólo P. cistoides se conocía para Chiapas (Villaseñor, 2016). Las características morfométricas de cada especie permiten distinguir a cada una de ellas.

Piriqueta mexicana fue descrita en 1987 con base en ejemplares colectados en Michoacán (Fryxell y Koch, 1987). La especie puede identificarse por su follaje extendido, pedúnculos largos y rectos, presencia de tricomas glandulares en tallo, hojas y peciolo, corola color durazno y sus frutos esféricos tuberculados. Desde su descripción se han colectado individuos en los estados de Michoacán y Guerrero relativamente cercanas a la costa del Pacífico, como puede observarse en la figura 1. Según la base de datos del Sistema Nacional de Información sobre Biodiversidad de México (CONABIO, 2018) aparece un registro en San Luis Potosí; sin embargo, no pudimos corroborarlo por la carencia de datos de colecta, aunado a que la altitud sobrepasa el rango frecuente de la especie, que va de 250 a 750 m (Arbo, 1995). Los ejemplares de P. mexicana de Chiapas se asemejan mucho al holotipo; coinciden morfológicamente con los ejemplares de la flora de Guerrero (Lozada, 2010) y con lo descrito por Arbo (1995), principalmente en la coloración de los pétalos, forma de las hojas, frutos y semillas, además de presentar tricomas en tallo, peciolo, hojas, pedúnculo floral y cáliz.

Con este nuevo registro se amplía el rango de distribución de Piriqueta mexicana, cuya presencia en Chiapas representa el límite sur de la especie y se documenta que la floración y fructificación ocurre durante un mayor intervalo (junio a diciembre) respecto a lo descrito por Arbo (1995) y
Lozada (2010), quienes señalan que la floración y fructificación ocurren de noviembre-diciembre.

Algunas explicaciones del porqué esta especie no había sido colectada en Chiapas pueden referirse a que es una planta poco llamativa mientras no presenta floración; es poco frecuente y tiene áreas de distribución restringida, por lo que pasa desapercibida a simple vista. Un esfuerzo más exhaustivo en el área ha permitido su detección. Con esto confirmamos la importancia de realizar colectas botánicas que formen parte de las colecciones museológicas (herbarios). Aunque recientemente hay interés por realizar registros fotográficos de varios taxa como parte de las iniciativas internacionales de ciencia ciudadana (p. ej. Naturalista, 2019), éstos no reemplazan el valor de los ejemplares botánicos, pero ayudan a preservar algunas características de forma y color de estructuras que se pierden al ser herborizadas. Ambas estrategias son complementarias; utilizadas en conjunto pueden asegurar de forma más efectiva que la información final satisfaga el interés de un mayor espectro de usuarios.

Este hallazgo resalta la importancia de las Áreas Naturales Protegidas como refugios efectivos para la permanencia de especies poco conocidas, por lo que se reconocen como áreas importantes para la conservación de la biodiversidad del país y a su vez denota la necesidad de continuar con las exploraciones botánicas.

\section{Contribución de autores}

DYSM realizó las colectas botánicas de Piriqueta mexica$n a$, revisó literatura especializada e identificó ejemplares y contactó a la especialista para corroborar la determinación taxonómica. ALGP revisó bases de datos y bibliografía especializada de $P$. mexicana. NRM revisó literatura especializada y ejemplares en línea de diferentes herbarios y realizó la traducción del resumen. Todos los autores contribuyeron en la redacción y revisión del manuscrito final.

\section{Financiamiento}

Este estudio fue apoyado por la Secretaría de Medio Ambiente e Historia Natural (SEMAHN), quien financió las salidas de campo al Área Natural Protegida "La Concordia Zaragoza" a través del proyecto "Monitoreo Biológico y Social de Áreas Naturales Protegidas". 


\section{Agradecimientos}

Los autores agradecen al director de las Áreas Naturales Protegidas, P. Sánchez Montero por las facilidades otorgadas para hacer uso de la información de las colectas de Piriqueta mexicana, a L. E. Gómez Pérez por el apoyo en la toma de fotografías del fruto y semillas y elaboración del mapa de distribución, a D. C. Gallegos López y M. A. García Jiménez por el apoyo en campo y a M. Ishiki Ishihara por el apoyo en la revisión del documento. Se le agradece a Ma. Mercedes Arbo por su apoyo en la revisión de Piriqueta mexicana.

\section{Literatura citada}

Arbo, M. M. 1995. Turneraceae: Parte I Piriqueta. Flora Neotropica 67: 1-156.

Areces-Berazain, F. y V. J. Vega-López. 2019. Hampea lanceolata (Malvaceae, Malvoideae), a new species from Chiapas and Oaxaca, Mexico. Phytotaxa 404(3): 121-126: DOI: https:// dx.doi.org/10.11646/phytotaxa.404.3.4

Breedlove, D. E. 1981. Flora of Chiapas. Part 1: Introduction to the Flora of Chiapas. California Academy of Sciences. San Francisco, USA. 35 pp.

Breedlove, D. E. 1986. Listados Florísticos de México IV. Flora de Chiapas. California Academy of Sciences e Instituto de Biología. Universidad Nacional Autónoma de México. México, D.F., México. 246 pp.

CONABIO. 2018. Sistema Nacional de Información sobre Biodiversidad. Registros de ejemplares. Comisión Nacional para el Conocimiento y Uso de la Biodiversidad. Ciudad de México, México. http://www.snib.mx/ejemplares/ descarga/ (consultado noviembre de 2019).

Dean, E., F. Archila, J. Poore, H. Kang, M. A. Anguiano-Constante, T. Starbuck y A. M. Rodríguez. 2019. Two new species of Lycianthes (Capsiceae, Solanaceae) from Mexico and Guatemala. Phytotaxa 409(5): 261-272: DOI: https://dx.doi. org/10.11646/phytotaxa.409.5.2

Fryxell, P. A. y S. D. Koch. 1987. New or Noteworthy Species of Flowering Plants from the Sierra Madre de Sur of Guerrero and Michoacán, Mexico. Aliso 11(4): 539-561.

García-Martínez, R., C. R. Beutelspacher-Baigts, R. CallejasPosada y G. Mathieu. 2018. Two new lithophytic species of the genus Peperomia (Piperaceae) from the state of Chiapas, Mexico. Phytotaxa 338(1): 109-116: DOI: https:// dx.doi.org/10.11646/phytotaxa.275.1.9
GBIF. 2019. Global Biodiversity Information Facility. https://www. gbif.org/ (consultado septiembre de 2019).

González-Espinosa, M., N. Ramírez-Marcial y L. Ruiz-Montoya (eds.). 2005. Diversidad biológica en Chiapas. Plaza y Valdés. México, D.F., México. 484 pp.

IBUNAM. 2019. Departamento de Botánica, Instituto de Biología (IBUNAM), Piriqueta mexicana Fryxell \& S.D. Koch, ejemplar de: Herbario Nacional de México (MEXU), Plantas Vasculares. Portal de Datos Abiertos, Universidad Nacional Autónoma de México. http://datosabiertos.unam.mx/ IBUNAM:MEXU:1433843 (consultado septiembre de 2019).

INAFED. 2010. Enciclopedia de Los Municipios y Delegaciones de México, Estado de Chiapas. http://www.inafed.gob.mx/ work/enciclopedia/EMM07chiapas/ (consultado octubre de 2019).

JSTOR. 2019. JSTOR Global Plants. https://plants.jstor.org/ (consultado noviembre de 2019).

Lot, A. y F. Chiang. 1986. Manual de herbario: administración y manejo de colecciones, técnicas de recolección y preparación de ejemplares botánicos. Consejo Nacional de la Flora de México. A.C. México, D.F., México. 142 pp.

Lozada, P. L. 2010. Turneraceae. Flora de Guerrero 43: 1-22.

Lozada-Pérez, L. 2019. A new species of Ilex (Aquifoliaceae) from Mexico. Phytotaxa 409(5): 296-300. DOI: https:// dx.doi.org/10.11646/phytotaxa.409.5.6

Martínez-Meléndez, N., M. Martínez-Meléndez y R. GarcíaMartínez. 2017. Lockartia hercodonta (Orchidaceae) en el volcán Tacaná, primer registro para la flora de Chiapas y México. Revista Mexicana de Biodiversidad 88(3): 761764. DOI: https://dx.doi.org/10.1016/j.rmb.2017.06.009

Naturalista. 2019. Comisión Nacional para el Conocimiento y Uso de la Biodiversidad. Cd. Mx., México. https://www. naturalista. $\mathrm{mx} /$

Ortiz-Díaz, J. J., I. Arnelas y J. P. Pinzón. 2016. Coccoloba tuni (Polygonaceae), a new species from Chiapas (Mexico). Phytotaxa 275(1):075-080:DOI:https://dx.doi.org/10.11646/ phytotaxa.275.1.9

Reyes-García, A. y M. Sousa S. 1997. Depresión Central de Chiapas, La Selva Baja Caducifolia. Listados Florísticos de México, XVII. Instituto de Biología, Universidad Nacional Autónoma de México (UNAM). Ciudad de México, México. 41 pp.

Rocha-Loredo, A. G., N. Ramírez-Marcial y M. González-Espinosa. 2010. Riqueza y diversidad de árboles del bosque tropical 
caducifolio en la Depresión Central de Chiapas. Boletín de la Sociedad Botánica de México 87: 89-103. DOI: https:// doi.org/10.17129/botsci.313

Ruiz-Sánchez, E., C. D. Tyrell, A. E. Ortíz-Rodríguez, V. Sosa y H. Gómez-Domínguez. 2019. A new species of Rhipidocladum (Poaceae: Bambusoideae: Arthrostylidiinae) from Mexico. Phytotaxa 420(4): 255-263: DOI: https://dx.doi. org/10.11646/phytotaxa.420.4.1

SEINEt. 2019. Southwest Environmental Information Network, SEINEt Arizona-New Mexico Chapter. http://swbiodiversity. org/ (consultado septiembre de 2019).

Torres-Colín, L. y L. O. Alvarado-Cárdenas. 2019. Desmodium amplistipulaceum (Leguminosae, Papilionoideae), a new species from northwest region of Chiapas, Mexico.
Phytotaxa 409(4): 215-221: DOI: https://dx.doi.org/10.11646/ phytotaxa.409.4.3

TROPICOS. 2019. Tropicos.org. Missouri Botanical Garden. http:// www.tropicos.org/Name/27902639 (consultado septiembreoctubre de 2019).

Vicente-Rivera, L. H., A. López-Cruz, B. N. Vicente-Rivera y M. A. Pérez-Farrera. 2019. Primer registro de Chiococca motleyana (Rubiaceae) para los bosques tropicales caducifolios de la Depresión Central de Chiapas, México. Acta Botanica Mexicana 126: e1392. DOI: https://dx.doi.org/10.21829/ abm126.2019.1392

Villaseñor R., J. L. 2016. Checklist of the native vascular plants of Mexico. Revista Mexicana de Biodiversidad 87(3): 559-902. DOI: https://dx.doi.org/10.1016/j.rmb.2016.06.017 